PROCEEDINGS OF THE

AMERICAN MATHEMATICAL SOCIETY

Volume 131, Number 12, Pages 3747-3755

S 0002-9939(03)06967-3

Article electronically published on February 14, 2003

\title{
PRINCIPAL EIGENVALUES FOR INDEFINITE WEIGHT PROBLEMS IN ALL OF $\mathbb{R}^{d}$
}

\author{
N. BEJHAJ RHOUMA
}

(Communicated by Juha M. Heinonen)

\begin{abstract}
We show the existence of principal eigenvalues of the problem $-\triangle u=\lambda g u$ in $\mathbb{R}^{d}$ where $g$ is an indefinite weight function. The existence of a continuous family of principal eigenvalues is demonstrated. Also, we prove the existence of a principal eigenvalue for which the principal eigenfunction $u \rightarrow 0$ at $\infty$.
\end{abstract}

\section{INTRODUCTION}

In this paper, we consider the following eigenvalue problem with indefinite weight:

$$
\begin{cases}\triangle u+\lambda g u=0 & \text { in } \mathbb{R}^{d} \text { in the distributional sense, } \\ u>0 & \text { on } \mathbb{R}^{d}\end{cases}
$$

for the case $d \geq 3$, where $g$ is a function in $K_{d}^{\text {loc }}\left(\mathbb{R}^{d}\right)$ that changes sign (i.e. $g$ is an indefinite weight). A principal eigenvalue of $(P)$ is a positive constant $\left(\lambda_{0}\right)$ for which $(P)$ has a positive solution for $\lambda=\lambda_{0}$.

Recently, a number of authors have investigated the existence of principal eigenvalues for $(P)$.

Brown, Cosner and Fleckinger in 5] showed that if $d \geq 3$ and $g$ is negative and bounded away of from 0 near $\infty$, then $(P)$ has a principal eigenvalue. Brown and Tertikas in [6] improved the result in [5] if $g^{+}=\max \{g, 0\}$ has a compact support. When $g$ is bounded and $g^{+} \in L^{\frac{d}{2}}\left(\mathbb{R}^{d}\right)$, the existence of one eigenvalue and infinitely many other eigenvalues was proved by Allegretto in [1]. Zhiren Jin in [10] considered the case when $g$ is locally Hölder continuous on $\mathbb{R}^{d}$. The author proved that if $d \geq 3$, $g\left(x_{0}\right)>0$ for some $x_{0} \in \mathbb{R}^{d}$ and if $\int_{\mathbb{R}^{d}}\left|g^{+}(y)\right|^{\frac{d}{2}} d y<\infty$, then there exists a continuous family of principal eigenvalues for the problem $(P)$. Moreover, the author showed that if in addition there exists $p>\frac{d}{2}$ such that $\int_{\mathbb{R}^{d}}|g(y)|^{p}\left(1+|y|^{2}\right)^{2 p-d} d y<\infty$, then $(P)$ has a principal eigenvalue $\left(\lambda_{0}\right)$ and a positive eigenfunction $u(x)$ such that $u(x)\|x\|^{d-2} \rightarrow c_{0}$ for a nonnegative constant $c_{0}$.

In our case, we do not give any assumption on the continuity and boundedness for $g$ and we will give a generalisation of the results cited above. In this paper, we assume that $g^{+} \not \equiv 0$, and we shall prove the following main results.

Received by the editors November 20, 2001 and, in revised form, June 25, 2002.

2000 Mathematics Subject Classification. Primary 31B20, 35J25, 35P05.

Key words and phrases. Indefinite weight, eigenvalue, Kato-class, Green function.

(C)2003 American Mathematical Society 
Theorem 1.1. Let $g \in K_{d}^{L o c}\left(\mathbb{R}^{d}\right)$ and let $G(x, y)$ denote the Green function on $\mathbb{R}^{d}$. If

$$
\left\|g^{+}\right\|_{\mathbb{R}^{d}}=\sup _{x \in \mathbb{R}^{d}} \int_{\mathbb{R}^{d}} G(x, y) g^{+}(y) d y<\infty,
$$

then there exists $\lambda^{*}>0$ such that for all $0<\lambda \leq \lambda^{*}$ there exists a positive continuous solution for the problem $(P)$.

Theorem 1.2. Let $g$ be in the Kato class $K_{d}\left(\mathbb{R}^{d}\right)$ such that $g^{+} \in L^{s}\left(\mathbb{R}^{d}\right)$ for $0<s<\frac{d}{2}$. Then, there exists $\lambda^{*}>0$ such that for all $0<\lambda \leq \lambda^{*}$ there exists a continuous positive solution for the problem $(P)$.

Remark 1.1. Note that if $g$ is bounded, then $g \in K_{d}\left(\mathbb{R}^{d}\right)$.

Theorem 1.3. Let $g \in K_{d}^{\operatorname{Loc}}\left(\mathbb{R}^{d}\right)$ such that

$$
\left\{\begin{array}{l}
\left\|g^{+}\right\|_{\mathbb{R}^{d}}<\infty \\
\lim _{\|x\| \rightarrow \infty}\left(\int_{\mathbb{R}^{d}} \frac{g^{+}(y)}{\|x-y\|^{d-2} d y}\right)=0 .
\end{array}\right.
$$

Then, $(P)$ has a principal eigenvalue $\lambda^{*}>0$ such that the corresponding eigenfunction $u$ satisfies $\lim _{\|x\| \rightarrow \infty} u(x)=0$.

Remark 1.2. Note that the conditions of Theorem 1.3 are less restrictive than the conditions of Zhiren Jin [10], where the author imposed that $g$ is locally Hölder continuous such that $g^{+} \in L^{\frac{d}{2}}$ and

$$
\int_{\mathbb{R}^{d}}|g(y)|^{p}\left(1+\|y\|^{2}\right)^{2 p-d} d y<\infty
$$

for some $p>\frac{d}{2}$. Indeed, any function in $L^{p}\left(p>\frac{d}{2}\right)$ is in $K_{d}\left(\mathbb{R}^{d}\right)$ and we will show in Proposition 3.4 that any function which satisfies the condition $\left(G^{\prime \prime}\right)$ lies in $L^{s}\left(\mathbb{R}^{d}\right)$ for some $s<\frac{d}{2}$ and hence satisfies the condition $(G)$. Moreover, we show the following general statement:

Theorem 1.4. Let $g$ be in the Kato class $K_{d}\left(\mathbb{R}^{d}\right)$ such that $g^{+} \in L^{q}$ for some $q<\frac{d}{2}$. Then the result of Theorem 1.3 holds.

Theorem 1.5. Let $g \in K_{d}^{\text {Loc }}\left(\mathbb{R}^{d}\right)$ such that $g^{+}$is a Green tight function in $\mathbb{R}^{d}$; namely, $g^{+}$is a Borel measurable function in $\mathbb{R}^{d}$ satisfying that

$$
\text { The family }\left\{\frac{g^{+}(.)}{\|\cdot-y\|^{d-2}}\right\} \text { is uniformly integrable }
$$

over $\mathbb{R}^{d}$ with the parameter $y \in \mathbb{R}^{d}$. Then $(P)$ has a principal eigenvalue $\lambda^{*}$ and a positive eigenfunction $u$ such that $\lim _{\|x\| \rightarrow \infty} u(x)=0$.

Moreover, we prove the following statement:

Corollary 1.1. We suppose that $g^{+}(x) \leq \frac{1}{\|x\|^{\alpha}}$ for $\|x\|$ large and some $\alpha>2$. Then there exists $\lambda^{*}$ such that the problem $(P)$ has a positive continuous eigenfunction such that $|u(x)| \leq c\|x\|^{2-d}$ for large $\|x\|$. 


\section{Preliminary}

Next, we recall from [2] the following definition:

Definition 2.1. A function $V$ is said to be in $K_{d}^{\text {loc }}\left(\mathbb{R}^{d}\right)$ if and only if, for every $R \geq 0$,

$$
\lim _{r \rightarrow 0}\left(\sup _{\|x\| \leq R} \int_{\|x-y\| \leq r} G(x, y)|V(y)| d y\right)=0 .
$$

A function $V$ is said to be in $K_{d}\left(\mathbb{R}^{d}\right)$ if and only if

$$
\lim _{r \rightarrow 0}\left(\sup _{x \in \mathbb{R}^{d}} \int_{\|x-y\| \leq r} G(x, y)|V(y)| d y\right)=0 .
$$

$G(x, y)$ is the Green function associated to the Laplace operator and $d y$ is the Lebesgue measure on $\mathbb{R}^{d}$.

Definition 2.2. For a bounded domain $\Omega$ in $\mathbb{R}^{d}$ let $G^{\Omega}(x, y)$ be the Green function defined on $\Omega \times \Omega$. We define the kernel associated to $V$ by

$$
K_{\Omega}^{V}=\int_{\Omega} G^{\Omega}(., y) V(y) d y
$$

and for every measurable function $g$, we define

$$
K_{\Omega}^{V} g=K_{\Omega}(V g)=\int_{\Omega} G^{\Omega}(., y) V(y) g(y) d y .
$$

In this paper, we say that $u=0$ on $\partial \Omega$ if $u\left(x_{n}\right) \rightarrow 0$ for every regular sequence $\left(x_{n}\right)$ in $\Omega$. Particularly, if $\Omega$ is regular, then $u\left(x_{n}\right) \rightarrow 0$ for every sequence $\left(x_{n}\right)$ converging to $z \in \partial \Omega$.

As in [4, we denote by $S_{b}^{V}(\Omega)$ the set of bounded functions $u$ such that $u+K_{\Omega}^{V} u$ is a superharmonic function in $\Omega$. If $V=0$, we will note $S_{b}^{V}(\Omega)=S_{b}(\Omega)$. Next, we recall the following definition (see [8]).

Definition 2.3. We say that the operator $I+K_{\Omega}^{V}$ is positive-invertible if the operator $I+K_{\Omega}^{V}: B_{b}(\Omega) \rightarrow B_{b}(\Omega)$ is invertible and for every function $s \in S_{b}^{+}(\Omega)$, we have $\left(I+K_{\Omega}^{V}\right)^{-1} s \geq 0$.

Without loss of generality, set $g=g_{1}-g_{2}$ with $g_{2}>0, g_{1}>m>0$.

Since $K g_{1}$ is a strict potential in $\Omega$, then by Theorem 4.1 in [8], for any $\lambda>0$ there exists a unique principal eigenvalue $\zeta(\lambda, \Omega)>0$ and a continuous eigenfunction $u_{\lambda}>0$ on $\Omega$ such that

$$
\Xi(\lambda, \Omega) \begin{cases}\triangle u_{\lambda}-\lambda g_{2} u_{\lambda}+\zeta(\lambda, \Omega) g_{1} u_{\lambda}=0 \text { in } \Omega \text { in the distributional sense, } \\ u_{\lambda}>0 & \text { in } \Omega, \\ u_{\lambda}=0 & \text { on } \partial \Omega .\end{cases}
$$

Using a result in [3] the function $\lambda \rightarrow \zeta(\lambda, \Omega)$ is continuous and for some $0<$ $\lambda<\mu$, we have $\zeta(\lambda, \Omega)-\lambda>0>\zeta(\mu, \Omega)-\mu$, whence $\zeta(\lambda, \Omega)=\lambda$ for some $\lambda$. If $\lambda(\Omega)=\inf \{\lambda>0: \zeta(\lambda, \Omega)=\lambda\}$, then $\lambda(\Omega)$ is a principal eigenvalue for

$$
E(\lambda, \Omega)\left\{\begin{array}{l}
\triangle u+\lambda g u=0 \text { in } \Omega \text { in the distributional sense, } \\
u>0 \text { in } \Omega \\
u=0 \text { in } \partial \Omega
\end{array}\right.
$$


Remark 2.1. The map $\lambda \rightarrow \lambda(\Omega)$ is decreasing. Indeed, let $\Omega_{1} \subset \Omega$ and set $\alpha=$ $\lambda\left(\Omega_{1}\right), \beta=\lambda(\Omega)$. Then, using Theorem 3.5 in [8], we obtain $\alpha=\zeta\left(\alpha, \Omega_{1}\right) \geq \zeta(\alpha, \Omega)$. Since for $\lambda$ small we have $\lambda<\zeta(\lambda, \Omega)$, we get the existence of $\omega \leq \alpha$ such that $\omega=\zeta(\omega, \Omega)$ which yields that $\beta \leq \alpha$.

\section{Construction of solutions of $(P)$}

Let $g=g^{+}-g^{-}$where $g^{+}(x)=\max \{g(x), 0\}$ and $g^{-}(x)=\max \{-g(x), 0\}$. We suppose that $\left\|g^{+}\right\|_{\mathbb{R}^{d}}<\infty$. Note that if $G^{\Omega}$ denotes the Green function on $\Omega$, then

$$
K_{\Omega} g^{+}=\int_{\Omega} G^{\Omega}(., y) g^{+}(y) d y \leq c(d)\left\|g^{+}\right\|_{\mathbb{R}^{d}}
$$

where $c(d)$ is a constant depending only on the dimension $d$.

We see that if $u_{\lambda}$ is a solution of $E(\lambda, \Omega)$ with $\left\|u_{\lambda}\right\|_{\infty}=1$, then

$$
\lambda(\Omega)=\frac{u_{\lambda}+\lambda(\Omega) K_{\Omega} g^{-}(u)}{K_{\Omega} g^{+}(u)} \geq \frac{u_{\lambda}}{c(d)\left\|g^{+}\right\|_{\mathbb{R}^{d}}} .
$$

Hence

$$
\lambda(\Omega) \geq \frac{1}{c(d)\left\|g^{+}\right\|_{\mathbb{R}^{d}}} .
$$

By Remark 2.1 since the map $\Omega \rightarrow \lambda(\Omega)$ is decreasing, then

$$
\lambda^{*}=\inf _{\Omega \subset \mathbb{R}^{d}} \lambda(\Omega)>0 .
$$

3.1. Proof of Theorem 1.1. Next, let $0<\mu \leq \lambda^{*}$. Then for all bounded domains $\Omega \subset \mathbb{R}^{d}$ we have $\mu<\lambda(\Omega)$.

Next, we claim that $\mu<\zeta(\mu, \Omega)$. Indeed, assume that $\mu>\zeta(\mu, \Omega)$. By using that for $\lambda$ small we have $\lambda<\zeta(\lambda, \Omega)$, we get the existence of $\lambda \in] 0, \mu[$ such that $\lambda=\zeta(\lambda, \Omega)$ which is impossible by the definition of $\lambda(\Omega)$. Hence, by Theorem 3.8 in [8], the operator $\left(I-\mu K_{\Omega}^{g}\right)$ is positive-invertible and for every $f \in C(\partial \Omega)$ there exists a function $u_{f}$ satisfying

$$
\begin{cases}\triangle u_{f}+\mu g u_{f}=0 & \text { in } \Omega \\ u_{f}=f & \text { on } \partial \Omega .\end{cases}
$$

Moreover $u_{f}>0$ on the set $\{f>0\}$.

Let $B_{n}$ be the ball centered at origin with radius $n, n=1,2, \ldots$. Then for each $n \in \mathbb{N}^{*}$, the boundary value problem

$$
\begin{cases}\triangle u_{n}+\mu g u_{n}=0 & \text { in } B_{n}, \\ u_{n}=n & \text { on } \partial B_{n}\end{cases}
$$

has a solution $u_{n}>0$ on $B_{n}$. We normalize $u_{n}(x)$ by setting $u_{n}(0)=1$. Then

$$
\left\{\begin{array}{l}
\Delta_{n}+\mu g u_{n}=0 \text { in } B_{n}, \\
u_{n}>0 \\
u_{n}(0)=1 .
\end{array}\right.
$$

By Harnack's inequality for positive solutions of elliptic equations (see 2]), we see that for any compact set $\Omega$ on $\mathbb{R}^{d}$, there are constants $N$ and $M$ (where $M$ depends only on $\Omega, \mu$ and $g, N$ depends only on $\Omega$ ) such that

$$
0<u_{n} \leq M \text { on } \Omega \text { for } n \geq N \text {. }
$$


Then it is clear that $\left(u_{n}\right)_{n}$ has a subsequence which converges to a continuous nonnegative function $u$ on any compact subset of $\mathbb{R}^{d}$.

Therefore

$$
\begin{cases}\triangle u+\mu g u=0 & \text { in } \mathbb{R}^{d} \\ u \geq 0 & \text { on } \mathbb{R}^{d} \\ u(0)=1 & \end{cases}
$$

Now, by an application of the minimum principle in [7], we get that $u>0$ in every bounded domain of $\mathbb{R}^{d}$ and hence $u>0$ on $\mathbb{R}^{d}$.

3.2. Proof of Theorem 1.2. Since $g^{+} \in K_{d}\left(\mathbb{R}^{d}\right)$, then there exists $\delta>0$ such that

$$
\sup _{x \in \mathbb{R}^{d}} \int_{\|x-y\| \leq \delta} \frac{g^{+}(y)}{\|x-y\|^{d-2}} d y \leq 1 .
$$

Hölder's inequality implies

$$
\begin{aligned}
\int_{\mathbb{R}^{d}-B(x, \delta)} \frac{g^{+}(y)}{\|x-y\|^{d-2}} d y & \leq\left\|g^{+}\right\|_{s}\left(\int_{\mathbb{R}^{d}-B(x, \delta)} \frac{1}{\|x-y\|^{(d-2) s^{\prime}}} d y\right)^{\frac{1}{s^{\prime}}} \\
& =\left|B_{1}\right|\left\|g^{+}\right\|_{s}\left(\int_{\delta}^{\infty} r^{d-1-s^{\prime}(d-2)} d s\right)^{\frac{1}{s^{\prime}}} \\
& =\left|B_{1}\right|\left\|g^{+}\right\|_{s} \delta^{2-\frac{d}{s}} .
\end{aligned}
$$

The result now follows from Theorem 1.1

3.3. Proof of Theorem 1.3. For each $k \in \mathbb{N}^{*}$, let us denote by $\lambda_{k}=\lambda\left(B_{k}\right)$ and by $\omega_{k}$ the principle eigenfunction to the problem

$$
E\left(\lambda, B_{k}\right) \quad\left\{\begin{array}{l}
\triangle u+\lambda_{k} g u=0 \text { in } B_{k}, \\
u>0 \quad \text { on } B_{k}, \\
u=0 \quad \text { on } \partial B_{k} .
\end{array}\right.
$$

The functions $\omega_{k}$ are chosen such that $\left\|\omega_{k}\right\|_{\infty}=1$.

Next, let $\bar{\omega}=\lambda_{1} \int_{\mathbb{R}^{d}} G(x, y) g^{+}(y) d y$. Thus

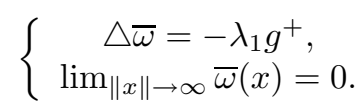

By Remark 2.1 the sequence $\lambda_{k}$ is decreasing. Hence on $B_{k}$, we have

$$
\begin{aligned}
\triangle\left(\omega_{k}-\bar{\omega}\right) & =-\lambda_{k} g \omega_{k}+\lambda_{1} g^{+} \\
& =\lambda_{1} g^{+}\left(1-\omega_{k}\right)+\lambda_{k} g^{-} \omega_{k}+\left(\lambda_{1}-\lambda_{k}\right) g^{+} \omega_{k} \\
& \geq 0 .
\end{aligned}
$$

Since $\omega_{k} \leq \bar{\omega}$ on $\partial B_{k}$, we get $\omega_{k} \leq \bar{\omega}$ on $B_{k}$. Thus, we can choose a subsequence of $\left(\omega_{k}\right)_{k}$ which converges uniformly on every compact of $\mathbb{R}^{d}$ to a nonnegative function $w$ such that

and satisfying

$$
\triangle \omega+\lambda^{*} g \omega=0 \quad \text { in } \mathbb{R}^{d}
$$

$$
0 \leq \omega \leq \bar{\omega}
$$

Since $g^{+}$satisfies the condition $(G)$, we get that $\lim _{\|x\| \rightarrow \infty} \bar{\omega}=0$. 
Next, we prove the following lemma.

Lemma 3.1. $\omega>0$ on $\mathbb{R}^{d}$.

Proof. Let $x_{k} \in B_{k}$ such that $\omega_{k}\left(x_{k}\right)=1$ and suppose that $\left\|x_{k}\right\| \rightarrow \infty$. Thus, we get

$$
1=\omega_{k}\left(x_{k}\right) \leq \bar{\omega}\left(x_{k}\right) \rightarrow 0 .
$$

Consequently, we can assume that there exists $x_{0} \in \mathbb{R}^{d}$ such that $x_{0}=\lim _{k \rightarrow \infty} x_{k}$. Let $k_{0}$ be such that $x_{k} \in B_{k_{0}}$ for all $k \in \mathbb{N}$. Since

$$
\omega_{k}=\lambda_{k} \int_{B_{k}} G^{B_{k}}(\cdot, y) g(y) \omega_{k}(y) d y
$$

we conclude that the family $\left\{\omega_{k}: k \in \mathbb{N}\right\}$ is equicontinuous and hence

$$
\omega\left(x_{0}\right)=\lim _{k \rightarrow \infty} \omega_{k}\left(x_{k}\right)=1 .
$$

Then, it follows that $\omega>0$ on $\mathbb{R}^{d}$.

3.4. Proof of Theorem 1.4. We recall the following theorem (Theorem 1.8 in [12]).

Theorem 3.2. Let $\mu$ be a positive Radon measure on $\mathbb{R}^{d}$ and let $f$ be nonnegative and $\mu$-measurable. Then

$$
\int_{\mathbb{R}^{d}} d \mu=\int_{0}^{\infty} \mu\{x: f(x)>t\} d t .
$$

Thus, we get the following lemma.

Lemma 3.3. Let $\mu$ be a positive Radon measure on $\mathbb{R}^{d}$ and $x \in \mathbb{R}^{d}$. Then

$$
\int_{\|x-y\| \geq \eta} \frac{d \mu(y)}{\|x-y\|^{d-2}} d y \leq(d-2) \int_{\eta}^{\infty} r^{1-d} \mu(B(x, r) d r .
$$

Proof. Using Fubini's Theorem and Theorem 3.2 we obtain

$$
\begin{aligned}
\int_{\|x-y\| \geq \eta} \frac{d \mu(y)}{\|x-y\|^{d-2}} d y & =(d-2) \int_{0}^{\infty} r^{1-d} \mu\left(B(x, r) 1_{\{\|x-y\| \geq \eta\}}\right) d r \\
& \leq(d-2) \int_{\eta}^{\infty}\left(\int_{\|x-y\|<r} r^{1-d} d \mu(y)\right) d r \\
& \leq(d-2) \int_{\eta}^{\infty} r^{1-d} \mu(B(x, r) d r .
\end{aligned}
$$

Next, we give the proof of Theorem 1.4.

Since $g^{+} \in L^{q}\left(\mathbb{R}^{d}\right)$, there exists $k \geq 0$ such that for every $x \in \mathbb{R}^{d}$ and $r \geq 0$, we have

$$
\int_{B(x, r)} g^{+}(y) d y \leq k\left\|g^{+}\right\|_{q} r^{d \frac{q-1}{q}} .
$$


Let $0<\eta<M$ and $x \in \mathbb{R}^{d}$ such that $\|x\|>M$. Using the last lemma and (3.2) we get

$$
\begin{gathered}
\int_{\mathbb{R}^{d}} \frac{g^{+}(y)}{\|x-y\|^{d-2}} d y \leq \int_{\|x-y\| \leq \eta} \frac{g^{+}(y)}{\|x-y\|^{d-2}} d y+(d-2) \int_{\eta}^{\infty}\left(\int_{B(x, r)} g^{+}(y) d y\right) \frac{d r}{r^{d-1}} \\
\leq \int_{\|x-y\| \leq \eta} \frac{g^{+}(y)}{\|x-y\|^{d-2}} d y+C\left(\int_{\eta}^{M} \int_{B(x, r)} g^{+}(y) d y \frac{d r}{r^{d-1}}+\int_{M}^{\infty} r^{d \frac{q-1}{q}} \frac{d r}{r^{d-1}}\right) \\
\leq \int_{\|x-y\| \leq \eta} \frac{g^{+}(y)}{\|x-y\|^{d-2}} d y \\
\quad+C\left(\left(\eta^{2-\frac{d}{q}}-M^{2-\frac{d}{q}}\right)\left(\int_{\|y\| \geq\|x\|-M}\left(g^{+}\right)^{q}(y) d y\right)^{\frac{1}{q}}+\frac{1}{M^{\frac{d}{q}-2}}\right)
\end{gathered}
$$

for some positive constant $C$.

Finally, let $\epsilon>0$. We choose, then, $\eta$ and $M$ such that $C \frac{1}{M^{\frac{d}{q}-2}}<\frac{\epsilon}{3}$ and

$$
\sup _{x \in \mathbb{R}^{d}} \int_{\|x-y\| \leq \eta} \frac{g^{+}(y)}{\|x-y\|^{d-2}} d y<\frac{\epsilon}{3} .
$$

Let $A \geq 0$ such that

$$
C\left(\eta^{2-\frac{d}{q}}-M^{2-\frac{d}{q}}\right)\left(\int_{\|y\| \geq A}\left(g^{+}(y)\right)^{q} d y\right)^{\frac{1}{q}}<\frac{\epsilon}{3} .
$$

Thus, for $\|x\| \geq A+M$, we get

$$
C\left(\eta^{2-\frac{d}{q}}-M^{2-\frac{d}{q}}\right)\left(\int_{\|y\| \geq\|x\|-M}\left(g^{+}(y)\right)^{q} d y\right)^{\frac{1}{q}}<\frac{\epsilon}{3} .
$$

Hence, $g^{+}$satisfies the condition $(G)$.

Next, we prove the following statement

Proposition 3.4. Let $g$ be a measurable function such that $g^{+} \not \equiv 0$ and

$$
\int_{\mathbb{R}^{d}}|g(y)|^{p}\left(1+\|y\|^{2}\right)^{2 p-d} d y<\infty
$$

for some $p>\frac{d}{2}$. Then, $g$ is in the Kato-class $K_{d}\left(\mathbb{R}^{d}\right)$ and there exists $q<\frac{d}{2}$ such that $g^{+} \in L^{q}$.

Proof. By $\left(G^{\prime \prime}\right)$, we get that $g \in L^{p}$ for $p>\frac{d}{2}$, which implies by a result in 2] that $g \in K_{d}\left(\mathbb{R}^{d}\right)$. Now, since $p>\frac{d}{2}$, we get that $\frac{d p}{4 p-d}<\frac{d}{2}$. Let $\frac{d p}{4 p-d}<q<\frac{d}{2}$. Using the fact that $\frac{q}{p}<1$ and the Hölder inequality we get

$$
\begin{aligned}
\int_{\mathbb{R}^{d}}|g|^{q} & \leq\left(\int_{\mathbb{R}^{d}}|g|^{p}\left(1+\|y\|^{2}\right)^{2 p-d} d y\right)^{\frac{1}{p}}\left(\int_{\mathbb{R}^{d}} \frac{d y}{\left(1+\|y\|^{2}\right)^{\frac{(2 p-d) q}{p-q}}}\right)^{\frac{p-q}{p}} \\
& \leq C\left(\int_{0}^{\infty} \frac{r^{d-1} d r}{\left(1+r^{2}\right) \frac{(2 p-d) q}{p-q}}\right)^{\frac{p-q}{p}} .
\end{aligned}
$$


Using the assumptions on $p$ and $q$, we get that $g \in L^{q}$.

\subsection{Proof of Theorem 1.5.}

Definition 3.5. A Borel function $k$ in $\mathbb{R}^{d}$ is called Green-bounded if and only if

$$
\|k\|_{\mathbb{R}^{d}}=\sup _{x \in \mathbb{R}^{d}} \int_{\mathbb{R}^{d}} \frac{|k(y)|}{\|x-y\|^{d-2}} d y<\infty .
$$

Definition 3.6. A Borel function $k$ in $\mathbb{R}^{d}$ is called Green-tight if and only if

$$
k \in K_{d}^{L o c}\left(\mathbb{R}^{d}\right) \quad \text { and } \quad \lim _{M \rightarrow \infty}\left(\sup _{x \in \mathbb{R}^{d}} \int_{\|y\| \geq M} \frac{|k(y)|}{\|x-y\|^{d-2}} d y\right)=0 .
$$

Remark 3.1. Any Green-tight function is Green-bounded.

Remark 3.2. Note that if there exists $\alpha>2$ and $c \geq 0$ such that $|k(x)| \leq \frac{c}{\|x\|^{\alpha}}$ for $\|x\|$ large, then $k$ is Green-tight. In fact we prove the following statement.

Proposition 3.7. Let $k$ be a Borel function in $\mathbb{R}^{d}(d \geq 3)$. Suppose that $k$ is in $K_{d}^{L o c}$ and there exists a number $L$ and a positive function $\varphi(r)$ on $[L, \infty[$ with $\int_{L}^{\infty} \frac{\varphi(r)}{r} d r<\infty$ such that for all $\|x\| \geq L$

$$
|k(x)| \leq \frac{\varphi(\|x\|)}{\|x\|^{2}} .
$$

Then $k$ is Green-tight.

For the proof see [14.

3.6. Proof of Corollary 1.1, Let $\bar{\omega}$ be the function defined in the proof of Theorem 1.3 Thus, using the results of [11] if $g^{+}(x) \leq \frac{1}{\|x\|^{\alpha}}$ for $\|x\|$ large and some $\alpha>2$, then

$$
\bar{\omega}(x)=\lambda_{1} \int_{B_{1}} G(x, y) g^{+}(y) d y \leq C\|x\|^{2-d}
$$

for large $\|x\|$ where $C$ is a positive constant. Since the solution $\omega$ defined in the previous section is such that $\omega \leq \bar{\omega}$, we get the desired proof.

\section{REFERENCES}

[1] L. Allegretto: Principal eigenvalues for indefinite weight elliptic problems in $\mathbb{R}^{N}$. Proc. Amer. Math. Soc., 116 (1992), 701-706. MR 93a:35114

[2] M. Aizenman and B. Simon: Brownian motion and Harnack inequality for schrödinger operator. Comm. Pure Appl. Math., 35 (1982), 209-273. MR 84a:35062

[3] N. Belhaj Rhouma and M. Mosbah: On the existence of positive eigenvalues for linear and nonlinear equations with indefinite weight. Appl. Anal., 81 (2002), 615-625.

[4] A. Boukricha, W. Hansen and H. Hueber: Continuous of the generalized Schrödinger equation and perturbation of harmonic spaces. Expo. Math., 5 (1987), 97-135. MR 88g:31019

[5] K.J. Brown, C. Cosner and J. Flekinger: Principal eigenvalues for problems with indefinite weight functions on $\mathbb{R}^{N}$. Proc. Amer. Math. Soc., 109 (1990), 147-155. MR 90m:35140

[6] K.J. Brown and A. Tertikas: The existence of principal eigenvalues for problem with indefinite weight functions on $\mathbb{R}^{N}$. Proc. Royal Soc. Edinburgh., 123 A (1993), 561-569. MR 94i:35136

[7] W. Hansen: Valeurs propres pour l'opérateurs de schrödinger. Séminaire de Théorie de Potentiel 9. Lecture Notes in Math., 1393 (1989), 117-134. 
[8] W. Hansen and H. Hueber: Eigenvalues in Potential theory. J. Diff. Equ., 73 (1988), 133-152.

[9] P. Hess and T. Kato: On some linear and nonlinear eigenvalue problems with an indefinite weight functions. Comm. Par. Diff. Equ., 5, (1980). 999-1030. MR 81m:35102

[10] Z. Jin: Principal eigenvalues with indefinite weight functions. Trans. Amer. Math. Soc., 349 (1997), 1945-1959. MR 97h:35056

[11] Y.Li and W.M. Ni: On conformal scalar curvature equations in $\mathbb{R}^{N}$. Duke Math J., 57 (1988) 895-924. MR 90a:58187

[12] J. Maly and W.P. Ziemer: Fine Regularity of Solutions of Elliptic Partial Differential equations. Amer. Math. Soc., Mathematical Surveys and Monographs. V 51. MR 98h:35080

[13] A. Manes and A.M. Micheletti: Un' estesione delle teoria variaziaonale classica degli autovalori per operatori elliptici del secondo ordine. Boll. Un. Mat. Italiana., 7 (1973), 285-301. MR 49:9402

[14] Z. Zhao: On the existence of positive solutions of nonlinear elliptic equations. A probalistic potential theory approach. Duke. Math. J., 69, (2), (1993), 247-258. MR 94c:35090

Institut Préparatoire aux Études d'Ingénieurs de Tunis, 2, Rue Jawaherlel Nehru, 1008 Montfleury, Tunis, Tunisia

E-mail address: Nedra.BelHajRhouma@ipeit.rnu.tn 